\title{
An Object Detection and Scaling Model for Plastic Waste Sorting
}

\author{
Abhishek Padalkar ${ }^{1}$, Pramod Pathak ${ }^{2}$, Paul Stynes ${ }^{3}$ \\ \{x19221576@student.ncirl.ie ${ }^{1}$,pramod.pathak@ncirl.ie ${ }^{2}$,paul.stynes@ncirl.ie $\left.{ }^{3}\right\}$
}

National College of Ireland, Mayor Street, IFSC, Dublin 01, Ireland ${ }^{1}$

\begin{abstract}
Plastic waste sorting involves the separation of plastic into its individual plastic types. This research proposes an Object Detection and Scaling Model for plastic waste sorting to detect four types of plastics using the $\mathrm{WaDaBa}$ dataset. This research compares the Object Detection and Scaling Models Scaled-Yolov4 and EfficientDet. Results demonstrate that Scaled-Yolov4CSP outperforms the state of the art, Colour-Histogram based Canny-Edge-Gaussian Filter, by $21 \%$ accuracy.
\end{abstract}

Keywords: Plastic Waste Sorting, Object Detection and Scaling Model, Scaled-Yolov4, EfficientDet

\section{Introduction}

Recycling centers use mechanical and chemical technology for plastic waste sorting. Current research is focusing on image-based smart waste recycling $[1,2,3,4,5]$. Plastic waste is sorted into its individual types namely, Polyethylene Terephthalate (PET), High-Density Polyethylene (HDPE), Polypropylene (PP), Polystyrene (PS), Polyvinyl Chloride (PVC), and Low-Density Polyethylene (LDPE). Bobulski and Piatkowski (2018) performed image classification to classify PET and NonPET plastic [6]. However, the challenge is to sort all types of plastics with higher accuracy.

The aim of the research is to investigate to what extent an Object Detection and Scaling Models can precisely and accurately sort plastic waste.

The major contribution of this research is a novel Object Detection and Scaling Model that utilizes feature pyramid networks, that optimizes the accuracy of sorting plastic waste. Feature pyramid network in object detection combines lower level and higher-level convolution features. It is performed by having bottom-up and top-down convolution pathways, connected laterally, of input image in order to extract high semantic information with higher resolution. This lateral interconnections between bottom-up and top-down convolutions improves the model detection and prediction accuracy as well as ability to detect small objects better due to better feature extraction. 
In order to identify the optimal Object Detection and Scaling Model this research compares Scaled-Yolov4-CSP, Scaled-Yolov4-p7, EfficientDet-d0, and EfficientDetd7x models trained on plastic waste images from the $\mathrm{WaDaBa}$ dataset. Based on accuracy, mean average precision (mAP), f1-measure for each plastic type, train time, inference time, and model size, a model was selected for the Object Detection and Scaling Framework.

\section{Related Work}

Mechanical [7, 8] and chemical [9] recycling is the current approach for plastic waste sorting. Mechanical plastic sorting technologies can only detect PVC plastics and which may be inaccurately recognized. Black colored plastics, plastic films, and some colored plastic remain undetected. Chemical methods can only separate high-density polymers, and separate plastic with sizes between $2-4 \mathrm{~mm}$.

Smart waste segregation is a modern approach in waste management and recycling where waste is segregated or classified using computer vision and neural network-based models [1]. In waste management, it is necessary to segregate recyclable waste from non-recyclable waste. Smart waste sorting can be achieved using an object detection model and by engaging the householders using machine learning-based gamification recycling applications [2,5]. Further, a cloud-based smart waste segregation architecture can be built using an object detection model to sort recyclable waste [3]. The ability of Convolution Neural Networks (CNNs) to provide reliable accuracy, learn new unique and abstract features shows promise in the domain of computer vision $[10,11,12]$.

Bobulski (2018), proposes a plastic waste dataset called WaDaBa to classify PET plastic types from the rest of the plastic types [6]. The approach used a colour-histogram and Canny-edgegaussian filter. Results demonstrate an accuracy of $75.68 \%$. The color-histogram method fails to incorporate features like shape and texture, which can improve classification results. The proposed WaDaBa plastic waste dataset shows promise for research on plastic waste.

The object detection model in plastic sorting is used to detect multiple plastic waste types in a single image frame. Object detection models that have a scaling ability of its internal components namely, depth, width, resolution of convolution, feature pyramid networks and its final classification and regression are referred to as Object Detection and Scaling Models. Scaling up depth increases number of stages of convolutions, width and resolution increases ability to capture more fine-grained features in the image, whereas scaling feature pyramid networks, final classification and regression modules improves on the accuracy of the object detection model. Research indicates that Scaled models outperform non-Scaled Object Detection Models [13, 14].

Plastics have different visual properties namely, gloss, transparency, haze, color, and so on. Differentiating between similar-looking plastics can be challenging. CNNs consistently demonstrate better performance in extracting visual properties [15]. Deep CNNs can detect transparent object features better with an ability to distinguish between transparent overlapping objects and non-transparent ones with the same shape $[16,17,18]$.

In plastic waste sorting, there is no research demonstrating performance of object detection models on plastic waste dataset. Thus, an object detection model with an ability to sort plastic waste objects needs to be selected. 
The backbone network in object detection models can have different neural network architectures such as DenseNet, InceptionNet, CSPDarkNet53, EfficientNet, and so on. However, it is important to select the best performing neural network architecture to extract the optimal number of features in object detection. The DenseNet with $95 \%$ and Inception-ResNet with $87 \%$ accuracy perform well in waste image classification [1]. The current state-of-the-art image classification models, EfficientNet and CSPDarkNet53, perform better on the ImageNet with 90\% and $94.8 \%$ accuracy respectively $[19,20]$. These models are used as backbone network in EfficientDet and Scaled-Yolov4 respectively. Feature pyramid network in object detection enhances feature extraction that improves overall mAP [21]. And, performing scaling on the feature pyramid network and final image classification and bounding box regression modules in the model improves $\mathrm{mAP}$ in object detection even further [13].

In object detection, objects that fall in the $32 \times 32$ pixel or lower category are categorized as small objects [21]. Plastic waste can be of varied sizes such as small medium or large sizes. Traditional object detection models show poor performance on small objects [21, 22]. Whereas, current state-of-the-art object detection models, Scaled-Yolov4 and Efficient-Det, confirm an increase in the performance of small, medium as well as large objects with their backbone neural network architecture and feature pyramid network $[13,14]$. Scaled-Yolov4-P7 and EfficientDet-d7 achieved 55.5 and $53.7 \mathrm{mAP}$, respectively, on the COCO dataset.

In conclusion, mechanical spectroscopy-based plastic waste sorting techniques are costly, complex, non-flexible, whereas it is seen that object detection-based smart waste segregation are easy to handle and flexible to upgrade. However, computer vision-based plastic waste sorting in plastic recycling has been largely understudied. While Bobulski et al. investigated the potential of plastic waste classification using the image colour-histogram-based feature extraction method, their work was limited to lower feature extraction and classification accuracy [6]. It is seen that current state-ofthe-art object detection models, Scaled-Yolov4 and EfficientDet are efficient and have higher feature extraction capability for small, medium and large objects. Aiming to address this gap, this work investigates a novel object detection and scaling model approach with better feature extraction capability, because of feature pyramid networks, to solve plastic waste sorting including PET, HDPE, $\mathrm{PP}$, and PS types of plastics.

\section{Methodology}

This research methodology follows five steps, namely Data Collection, Pre-Processing, Transformation, Data Modelling Training and Inference, and Evaluation and Results, as shown in fig.1.

In the first step, Data Collection, the "WaDaBa" plastic dataset published by Bobulski and Piatkowski (2018) was collected from the official website [6]. ${ }^{1}$ The dataset consists of four thousand plastic waste images of 5 plastic types namely PET, HDPE, PP, PS, and "Other".

In the second step, Data Pre-Processing, the image dataset was first converted to an object detection dataset by manually creating bounding boxes for 4000 plastic waste images. Bounding boxes are rectangles that surrounds the object of interest. Auto-orientation was also applied as the

\footnotetext{
1"WaDaBa" Dataset: http://wadaba.pcz.pl/index.html
} 


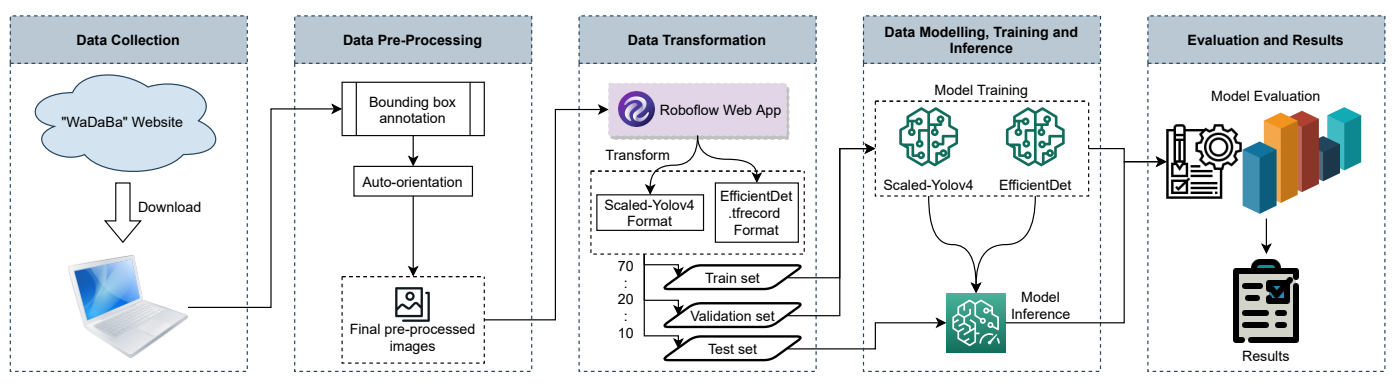

Fig. 1. Methodology

final pre-processing to the dataset.

In the third step, Data Transformation, the image dataset was transformed in the object detection model format of Yolo and EfficientDet. Using the Roboflow web app, the image dataset along with bounding boxes file was converted into Yolo bounding box format as class, centre $\mathrm{x}$, centre $\mathrm{y}$, width, height. Likewise, for the EfficientDet format was converted into a .tfrecord file with bounding boxes as xmax, xmin, ymax, ymin with a class label. Finally, the dataset was split into 70:20:10 ratio as train, validation, and test, respectively.

In the fourth step, Data Modelling and Training, state of the art object detection models, ScaledYolov4 and EfficientDet with their individual baseline and highest scaled models were trained on the transformed plastic dataset $[13,14]$. These models were pre-trained on the COCO dataset and then all the weights were finetuned thereafter on the plastic dataset. As discussed in previous section, object detection models have not been used on the plastic sorting domain. Furthermore, the above-mentioned models are selected to perform plastic sorting task because of their state of the art results in object detection based on generic comparison on COCO dataset. For Scaled-Yolov4, first, the baseline scaledyolov4-csp model was trained with batch size 16, followed by the scaled-yolov4p7 model with batch size 8. For the EfficientDet, first, efficientdet-d0 was trained with batch size 32 for faster training, and efficientdet-d7x was trained with batch size 8 . The batch size was reduced for both of the scaled models due to excessive memory usage while training. For smaller models, Scaled-Yolov4-CSP and Efficientdet-d0, larger batch size is used; whereas for larger models, ScaledYolov4-p7 and Efficientdet-d7, smaller batch size is used. The batch sizes selected differ according to the memory available to train the models to avoid running out of memory while training. All four models were trained for 100 epochs, and the learning rate for both EfficientDet models was set to 0.05 to obtain convergence. The batch-size were set by trial and error whereas the other hyperparameters, learning rate and number of epochs, were set to obtain convergence with no overtraining. The trained models were then used for inference on the test set.

In the fifth step, evaluation, the inference output of each model was evaluated based on mean average precision and accuracy. The model performance is compared based on training time, model size, and inference time. For each plastic-type, the model f1-score is also evaluated. The final results of each model are compared and visualized using the matplotlib python library. 


\section{Design and Implementation}

The architecture for plastic waste sorting is shown in fig.2. The WaDaBa test images are passed to the object detection and scaling models to make an inference in order to classify and detect the plastic object type [23]. The classification setup for the experiment performed was multiclass classification as the dataset utilized was a multi-class dataset. An output inference detection for plastic object images is generated, with a classified plastic object type along with the bounding box and classification accuracy.

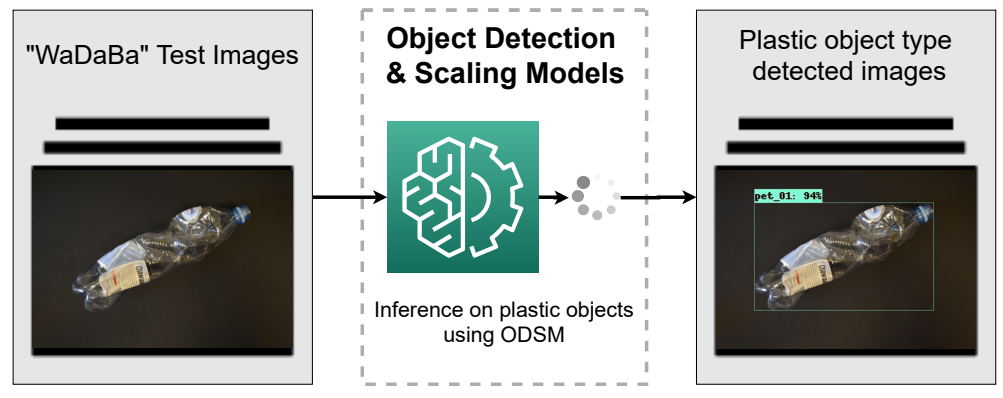

Fig. 2. Design architecture

The Object Detection and Scaling models were implemented on jupyter notebook. The WaDaBa dataset was uploaded to Labelbox. The Labelbox platform was used to create a plastic waste object detection dataset that adds bounding box annotations to the $\mathrm{WaDaBa}$ dataset. This final bounding box annotated dataset was exported as a .json file. The dataset is uploaded on Roboflow. Using Roboflow, the dataset are created and exported to the Object Detecion and Scaled Models. The generated dataset is uploaded on google drive and then imported to Google Colab Pro for model training. The Scaled-Yolov4 model is implemented in Pytorch. The EfficientDet model is implemented in Tensorflow [13, 14]. All experiments were performed on Google Colab with machines having Tesla P100 or V100 16GB graphics card with 32GB memory. A total of 4 models were finetuned on our plastic dataset, Scaled-Yolov4-CSP baseline model, Scaled-Yolov4 P7 scaled model, EfficientDet-d0 baseline model, and EfficientDet-d7x scaled model on the WaDaBa plastic dataset.

\section{Evaluation}

The aim of the experiment was to identify the optimal ODSM from EfficientDet-d0, EfficientDetd7x,Scaled-YoloV4-CSP, and Scaled-YoloV4-p7.

Table 1 display the results of the models based on their mAP, Class Prediction Accuracy and F1 Scores. The Class Prediction Accuracy scores are on the test set, while the mAP values are from the training set. The Class Prediction Accuracy confirms how accurately the model classifies plastic waste.The mAP values proves how precisely the model detects the object taking into account the bounding box edges around the objects for each class. The results indicate that the Scaled-Yolov4CSP model outperformed all the other models with $0.97 \mathrm{mAP}$ and $97 \%$ accuracy. It is seen that 
Scaled-Yolov4-CSP significantly outperformed the accuracy of $75.68 \%$ reported in the state of the art by Bobulski and Piatkowski (2018) [6].

A significant difference is seen in mAP for Scaled-Yolov4-p7 and EfficientDet-d7 $x$ as compared to their baseline models. As these models are very large in size with large number of parameters, the dataset size used to train them is small relatively that is suspected to give the displayed mAP scores. Whereas, the lighter baseline models performed well on the given dataset size having comparatively less parameters.

A comparison of all the trained models by f1-scores for each plastic type is shown in table 1 . All ODSMs achieved a high f1-score for PET plastic objects. Moreover, PP and PS plastic objects are less precisely detected than PET and HDPE plastic types.

Table 1: mAP, Class Accuracy and F1 score comparison of object detection models

\begin{tabular}{|c|c|c|c|c|c|c|}
\hline Model & mAP & Class Prediction Accuracy & \multicolumn{4}{|c|}{ F1-Scores } \\
\hline & & & PET & HDPE & PP & PS \\
\hline Scaled-Yolov4-CSP & 0.97 & $97 \%$ & 1.0 & 1.0 & 0.95 & 0.94 \\
\hline Scaled-Yolov4-p7 & 0.72 & $95 \%$ & 1.0 & 0.99 & 0.88 & 0.87 \\
\hline EfficientDet-d0 & 0.65 & $69 \%$ & 0.90 & 0.51 & 0.38 & 0.30 \\
\hline EfficientDet-d7x & 0.56 & $71 \%$ & 0.90 & 0.49 & 0.54 & 0.35 \\
\hline Colour-Histogram and Canny-edge-gaussian filter [6] & - & $75.68 \%$ & - & - & - & - \\
\hline
\end{tabular}

A comparison of all the trained models by training time and inference time is displayed in fig.3a. Training time is the total time consumed by the model to train over 100 epochs. Inference time is the time taken for the model to detect and classify the plastic object. Less time, for both training and inference, indicates better model performance. It is also seen that Scaled-Yolov4-CSP models consumed significantly less time to train and make an inference with $1.48 \mathrm{hrs}$ and $0.02 \mathrm{~s}$, respectively, with higher accuracy, mAP, and F1-score.

Model size comparison of all the trained models is shown in fig.3b. It is seen that EfficientDet models require significantly less hard drive space to store the model weights than Scaled-Yolov4. But, it is seen that Scaled-Yolov4-CSP uses only $420 \mathrm{mb}$ of drive space which is also less and comparatively better for an object detection model weights.

Scaled-Yolov4-CSP ODSM outperforms in plastic recognition than histogram Canny edge filter based study by roughly $21 \%$ accuracy on "WaDaBa" dataset.

\section{Conclusion and Future work}

The aim of the research is to investigate to what extent an Object Detection and Scaling Models can precisely and accurately sort plastic waste. This research demonstrates that Scaled-Yolov4-CSP outperforms Scaled-Yolov4-p7, EfficientDet-d0 and EfficientDet-d7x with accuracy of 97\%, mAP of $97 \%$, train time of $1.48 \mathrm{hrs}$, inference time of $0.02 \mathrm{~s}$ and $420 \mathrm{mb}$ model size. Object Detection and Scaling Models with feature pyramid networks outperform the state of the art that use Canny edge gaussian filter by $21 \%$ accuracy. This research shows the potential of using the ODSM ScaledYolov4-CSP for plastic waste sorting in practice. 


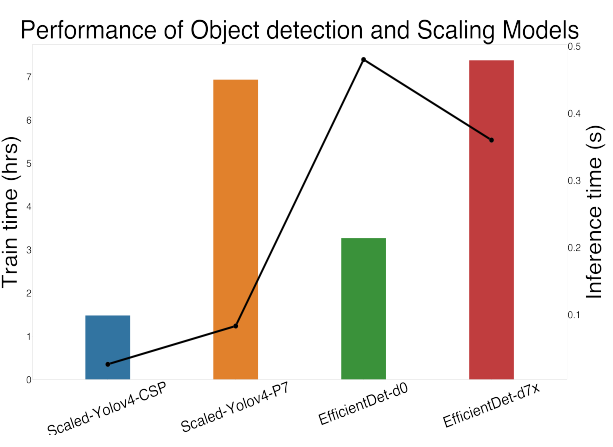

(a) Train vs Inference time

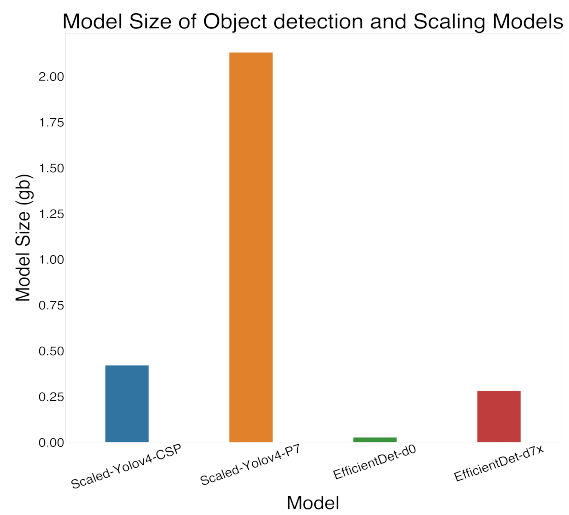

(b) Model Size

Fig. 3. Model Performance Summaries

This research detected plastic waste objects using feature pyramid networks as a feature enhancement. Future research could explore an object detection model that also utilizes contextual features to detect plastic waste objects.This work focused on PET, HDPE, PP, and PS plastic types. In future research "Other" and LDPE plastic waste types will be investigated.

\section{References}

[1] Bircanoğlu C, Atay M, Beşer F, Genç Kızrak MA. RecycleNet: Intelligent Waste Sorting Using Deep Neural Networks. 2018:1-7.

[2] Kumar S, Yadav D, Gupta H, Verma OP, Ansari IA, Ahn CW. A Novel YOLOv3 AlgorithmBased Deep Learning Approach for Waste Segregation: Towards Smart Waste Management. Electronics. 2021;10(1).

[3] Ziouzios D, Tsiktsiris D, Baras N, Dasygenis M. A Distributed Architecture for Smart Recycling Using Machine Learning. Future Internet. 2020;12(9).

[4] Peršak T, Viltužnik B, Hernavs J, Klančnik S. Vision-Based Sorting Systems for Transparent Plastic Granulate. Applied Sciences. 2020;10(12).

[5] Ekundayo O, Murphy L, Pathak P, Stynes P. An On-Device Deep Learning Framework to Encourage the Recycling of Waste. In: Arai K, editor. Intelligent Systems and Applications. Cham: Springer International Publishing; 2022. p. 405-17.

[6] Bobulski J, Piatkowski J. PET Waste Classification Method and Plastic Waste DataBase WaDaBa. 2018:57-64.

[7] Gundupalli SP, Hait S, Thakur A. A review on automated sorting of source-separated municipal solid waste for recycling. Waste Management. 2017;60:56-74. Special Thematic Issue: Urban Mining and Circular Economy.

[8] Hahladakis JN, Iacovidou E. An overview of the challenges and trade-offs in closing the loop of post-consumer plastic waste (PCPW): Focus on recycling. Journal of Hazardous Materials. 2019;380:120887. 
[9] Singh N, Hui D, Singh R, Ahuja IPS, Feo L, Fraternali F. Recycling of plastic solid waste: A state of art review and future applications. Composites Part B: Engineering. 2017; Volume 115:409-22.

[10] Howard AG, Zhu M, Chen B, Kalenichenko D, Wang W, Weyand T, et al. MobileNets: Efficient Convolutional Neural Networks for Mobile Vision Applications. CoRR. 2017;abs/1704.04861.

[11] Ren S, He K, Girshick R, Sun J. Faster R-CNN: Towards Real-Time Object Detection with Region Proposal Networks. IEEE Transactions on Pattern Analysis and Machine Intelligence. 2017;39(6):1137-49.

[12] Zhou Y, Liu L, Shao L, Mellor M. DAVE: A Unified Framework for Fast Vehicle Detection and Annotation. 2016:278-93.

[13] Tan M, Pang R, Le QV. EfficientDet: Scalable and Efficient Object Detection. In: Proceedings of the IEEE/CVF Conference on Computer Vision and Pattern Recognition (CVPR); 2020. .

[14] Wang CY, Bochkovskiy A, Liao HYM. Scaled-YOLOv4: Scaling Cross Stage Partial Network. In: Proceedings of the IEEE/CVF Conference on Computer Vision and Pattern Recognition (CVPR); 2021. p. 13029-38.

[15] Luo Q, Fang X, Su J, Zhou J, Zhou B, Yang C, et al. Automated Visual Defect Classification for Flat Steel Surface: A Survey. IEEE Transactions on Instrumentation and Measurement. 2020;69(12):9329-49.

[16] McDonnell MD, Moezzi B, Brinkworth RSA. Using Style-Transfer to Understand Material Classification for Robotic Sorting of Recycled Beverage Containers. 2019:1-8.

[17] Madessa AH, Dong J, Dong X, Gao Y, Yu H, Mugunga I. Leveraging an Instance Segmentation Method for Detection of Transparent Materials. In: 2019 IEEE SmartWorld, Ubiquitous Intelligence Computing, Advanced Trusted Computing, Scalable Computing Communications, Cloud Big Data Computing, Internet of People and Smart City Innovation; 2019. p. 406-12.

[18] Khaing MP, Masayuki M. Transparent Object Detection Using Convolutional Neural Network. In: Zin TT, Lin JCW, editors. Big Data Analysis and Deep Learning Applications. Singapore: Springer Singapore; 2019. p. 86-93.

[19] Wang CY, Liao HYM, Yeh IH, Wu YH, Chen PY, Hsieh JW. CSPNet: A New Backbone that can Enhance Learning Capability of CNN; 2019.

[20] Bochkovskiy A, Wang CY, Liao HYM. YOLOv4: Optimal Speed and Accuracy of Object Detection; 2020.

[21] Leng J, Ren Y, Jiang W, Sun X, Wang Y. Realize your surroundings: Exploiting context information for small object detection. Neurocomputing. 2021;433:287-99.

[22] Liu Y, Sun P, Wergeles N, Shang Y. A survey and performance evaluation of deep learning methods for small object detection. Expert Systems with Applications. 2021;172:114602.

[23] Padalkar A, Pathak P, Stynes P. An Object Detection and Scaling Model for Plastic Waste Sorting - Implementation Code; 2021. Available from: https://drive.google.com/drive/folders/16qk $C-Y V M 02 E g g a X h Q 5 B V 8 \operatorname{co3} 6 v 9 Z L j$ ?usp = sharing. 